\title{
Evaluación del desempeño académico en entornos virtuales utilizando el modelo PNL
}

\author{
Raquel Aguayo ${ }^{1}$, Carmen Lizarraga ${ }^{1}$, Yadira Quiñonez ${ }^{1}$ \\ raquelaguayog@uas.edu.mx; carmen.lizarraga@uas.edu.mx; yadiraqui@uas.edu.mx \\ ${ }^{1}$ Universidad Autónoma de Sinaloa, Facultad de Informática, Av. Leonismo Internacional S/N y Av. de los \\ Deportes, Ciudad Universitaria, 82000, Mazatlán, Sinaloa, México.
}

DOI: 10.17013/risti.41.34-49

\begin{abstract}
Resumen: En la actualidad, ha incrementado considerablemente los avances e innovaciones tecnológicas, lo que ha permitido el desarrollo de nuevas herramientas tecnológicasyentornos virtuales para optimizar el proceso deenseñanza-aprendizaje. Este trabajo se centra en evaluar el desempeño académico de los estudiantes de la Licenciatura en Gastronomía de la Universidad Autónoma de Sinaloa (UAS) en entornos virtuales, específicamente en la asignatura de estadística. La metodología aplicada fue cuasi-experimental con un enfoque longitudinal descriptivo mixto por encuesta. El análisis de contexto se realizó a través de un instrumento para recolectar información relacionada con el uso y manejo de herramientas tecnológicas. Además, se aplicó el modelo de Progrogramación Neurolingística (PNL) para conocer el estilo de aprendizaje predominante en los estudiantes. Finalmente, se analizó el desempeño académico de los estudiantes durante el desarrollo de dos unidades de aprendizaje. Este documento finaliza con una discusión crítica de los resultados obtenidos, las conclusiones y recomendaciones.
\end{abstract}

Palabras-clave: proceso de enseñanza aprendizaje; entornos virtuales; estilos de aprendizaje; rendimiento académico.

\section{Evaluation of academic performance in virtual environments using the NLP model}

Abstract: At present, it has considerably increased technological advances and innovations, which has allowed the development of new technological tools and virtual environments to optimize the teaching-learning process. This work focuses on evaluating the students' academic performance of the Bachelor of Gastronomy of the UAS in virtual environments, specifically in the subject of statistics. The applied methodology was quasi-experimental with a mixed descriptive longitudinal approach per survey. The context analysis was carried out through an instrument to collect information related to technological tools' use and management. Also, the NLP model was applied to know the predominant learning style in the students. Finally, the academic performance of the students was analyzed during the development of two learning units. This document ends with a critical discussion of the results obtained, the conclusions, and recommendations. 
Keywords: teaching-learning process; virtual environments; learning styles; academic performance.

\section{Introducción}

Actualmente, vivimos inmersos en la llamada sociedad de la información, debido a que el uso de la tecnología ha tenido un incremento considerable, esto se ve reflejado en la educación, donde el modelo tradicional ha ido evolucionado con el uso de las nuevas Tecnologías de la Información y de la Comunicación (TIC) (Seitebaleng, 2018), así como la aplicación de espacios educativos virtuales; que sin duda alguna han venido marcando otra era (Zambrano, 2020). Cuando se habla de tecnología se refiere a un conjunto de conocimientos y técnicas aplicadas, que facilitan la creación de herramientas y entornos virtuales para que los estudiantes puedan intercambiar y colaborar entre ellos, y de esta forma lograr la optimización del proceso de enseñanza aprendizaje (Lizarraga, 2017), el aprendizaje colaborativo es considerado una técnica de aprendizaje muy efectiva (Quiñonez, 2016). Por esta razón, el constante cambio tecnológico lleva a la sociedad a aplicar la tecnología en diversas áreas, tales como: la medicina, las comunicaciones, el medio ambiente, la industria y la educación (Srivastava, 2014). La tecnología aplicada a la educación es de gran ayuda para que los estudiantes lleven a cabo el proceso de enseñanza y aprendizaje de una manera favorable y autodidacta (Lekka, 2015).

Debido a la importancia y a las condiciones actuales de enseñanza en los diferentes niveles educativos, algunos autores (Hui, 2016) proponen aprovechar la situación actual de los estudiantes que han crecido bajo la influencia de la tecnología interactiva, creando comunidades del conocimiento a partir de aprendizaje combinado. Esto es, con el uso e incorporación de tecnologías emergentes se ha proporcionado a la humanidad canales nuevos de comunicación e inmensas fuentes de información que difunden modelos de comportamiento social, actitudes, valores y formas de organización, como resultado, se mejora la calidad en el proceso de enseñanza aprendizaje, los estudiantes adquieren más responsabilidad, mejoran las habilidades y destrezas tanto en el estudio individual como grupal (Lillejord, 2018).

Recientemente, la pandemia de COVID-19 obligó a las instituciones educativas de todos los niveles académicos a cambiar los entornos de aprendizaje, sin embargo, el principal inconveniente que tenían la mayoría de las instituciones era la falta de un plan de contingencia o la tecnología adecuada para realizar todos los cursos en línea. El autor Attallah (2020) presenta un concentrado de diferentes trabajos relacionados con la experiencia de algunas universidades post COVID-19, centrándose en la aplicación de entornos virtuales, destaca las ventajas y la idoneidad de las herramientas tecnológicas utilizadas en las actividades para el aprendizaje en línea.

Por otro lado, Kumar et al. (2020) presentan un trabajo relacionado con las herramientas de asistencia tecnológica que están disponibles para que se pueda impartir una educación de calidad en entornos en línea. Particularmente, se enfocan en los Seminarios Web (Webinars) como herramienta de enseñanza para que los profesores puedan llevar a cabo sus clases durante este período de COVID-19. Dentro de las plataformas utilizadas para los Webinars destacan las siguientes: Zoom (Zoom, 2021), GoToMeeting (Gotomeeting, 2021), GoToWebinar (GoToWebinar, 2021), Google Meet (Google Meet, 2021), Cisco 
Webex (Cisco Webex, 2021) y Microsoft Team (Microsoft Team, 2021). De cada una de estas plataformas han realizado una comparación con sus principales características tales como: versión gratuita disponible, número de participantes de la reunión, compartir pantalla, pizarra, grabación de las reuniones, seguridad (cifrado E2E), planes de pago, aplicación móvil, espacio de trabajo colaborativo, entre otras. Destacan la importancia de la exploración de diferentes plataformas, la capacitación de profesores y estudiantes, así como la realización de sesiones de demostración y la introducción de plataformas de evaluación.

Por lo tanto, de acuerdo con los trabajos mencionados anteriormente, este trabajo tiene como objetivo evaluar el desempeño académico de los estudiantes de Licenciatura en Gastronomía de la UAS en entornos virtuales, específicamente en la asignatura de estadística, utilizando el modelo PNL para conocer el estilo de aprendizaje predominante en los estudiantes y de esta forma estimular todos los canales de aprendizaje.

El resto de este artículo está organizado de la siguiente manera: en la Sección 2 se presenta el contexto teórico que fundamentan el proceso de la investigación en lo que se refiere a las TIC. La sección 3, describe la estrategia metodológica aplicada en este trabajo. En la Sección 4, se presentan los resultados obtenidos con el instrumento de contexto, resultados de la aplicación del modelo PNL y el análisis de rendimiento académico. Finalmente, la sección 5 presenta un resumen de las conclusiones obtenidas con la realización de este trabajo.

\section{Contexto teórico}

Un tema básico en el estudio del aprendizaje son sus teorías, las cuales han cambiado a lo largo de la historia, haciéndose presentes unas más que otras. Shuell, en 1986 menciona que aprender es un cambio perdurable de la conducta o en la capacidad de conducirse de manera dada como resultado de la práctica o de otras formas de experiencia. Basados en este concepto, se puede decir que el aprendizaje se presenta de manera empírica en todos los aspectos de la vida y empieza desde pequeños. Sin embargo, para la sociedad es importante que sus miembros estén completamente preparados para poder introducirse y desarrollarse satisfactoriamente en el campo laboral, y tener como resultado no sólo el desarrollo del individuo como profesionista sino también la sociedad de la que forma parte.

\subsection{Modelos educativos.}

Los modelos educativos surgen de corrientes de pensamiento y teorías, las cuales abordan distintos paradigmas. Cada modelo tiene su propio paradigma, que habla sobre la educación y por supuesto del proceso enseñanza-aprendizaje. Existen diferentes modelos educativos, en este trabajo se abordan 5 que sustentan ésta investigación.

- Modelo conductista: Según Minetti (2015) se basa en la relación que existe entre estímulo y reacción, donde el estímulo lo proporciona el docente de diferentes formas y las reacciones del estudiante se observan para posteriormente elegir el estímulo correcto, para que el estudiante reaccione de la manera esperada; esta teoría se basa en los estudios de Skinner (1997). 
- Modelo cognitivo: De acuerdo con Gómez \& Polanía (2008) el modelo se enfoca en las teorías de Dewey (1957) y Piaget (1999); quienes plantearon que la educación debe buscar que cada individuo acceda progresiva y secuencialmente a una etapa superior del desarrollo intelectual, en relación con las necesidades y condiciones particulares de cada uno, con el fin de constituir en la meta educativa.

- Modelo Psico-social: Este modelo se refiere al estudio sistemático de los componentes, procesos y resultados de las intervenciones y programas que implican el conocimiento a través de técnicas de evaluación, así como del estudio de la población estudiantil a la que se pretende evaluar, asesorar, dinamizar, planificar y programar, en bienestar al proceso de enseñanza aprendizaje.

- Modelo constructivista: Según Minetti (2015) este modelo es fundamentado principalmente por Vigotsky, Piaget y Ausubel, quienes definieron al constructivismo como la aplicación y disposición de diferentes métodos de apoyo que le permiten al alumno construir su propio saber. Según este modelo, cada persona construye su propia perspectiva del mundo que le rodea a través de sus propias experiencias y esquemas mentales desarrollados.

- Modelo conectivista: Gayol de Pallán (2016) menciona que este modelo es el producto del desarrollo de la informática. Siemens (2007) plantea que las redes de cualquier naturaleza ya sean informáticas o sociales pueden ser conectadas con un todo integrado y que al alterarse las partes se altera todo. El conectivismo es la integración de principios explorados por el caos, las redes, la complejidad y las teorías de auto-organización.

\subsection{Estilos de aprendizaje}

El término "estilo de aprendizaje" básicamente se refiere a la forma en que cada individuo recibe o comprende el conocimiento, y generalmente, es utilizado como su propio método o estrategia de aprendizaje (Pantoja, 2013). Dichos estilos de aprendizaje permiten optimizar el proceso de enseñanza para el docente y el de aprendizaje para el estudiante, ya que ayuda a comprender por qué algunas estrategias pueden funcionar perfectamente para un grupo de estudiantes y para otros no. Por esta razón, una vez que el docente conoce los estilos de aprendizaje que existen, puede identificar cuales predominan en el grupo y de esta forma poder implementar las estrategias didácticas más adecuadas que fortalezcan todos los canales de aprendizaje de los estudiantes (Bustos-Lopez, 20218). En la Tabla 1 se muestran algunos de los modelos de estilos de aprendizaje más conocidos y utilizados, según Gómez, Aduna \& García (2004), a través de los cuales se pueden entender los comportamientos diarios en el aula y la forma en que aprenden los alumnos y las acciones que resultan más eficaces.

\begin{tabular}{lll}
\hline Modelo & Estilo de aprendizaje & Características del modelo \\
\hline $\begin{array}{l}\text { Cuadrantes cerebrales } \\
\text { de Herrmann }\end{array}$ & $\begin{array}{l}\text { Cortical izquierdo, Límbico } \\
\text { izquierdo, Límbico derecho y } \\
\text { Cortical derecho }\end{array}$ & $\begin{array}{l}\text { Representan cuatro formas distintas de } \\
\text { operar, de pensar, de crear, de aprender y, } \\
\text { en suma, de convivir con el mundo }\end{array}$ \\
\hline \multirow{2}{*}{ Felder y Silverman } & $\begin{array}{l}\text { Sensitivo/intuitivo, } \\
\text { Visual/verbal, Inductivos/ } \\
\text { Deductivos. Secuenciales/ } \\
\text { globales, Activos/reflexivos }\end{array}$ & $\begin{array}{l}\text { Conciben los estilos de aprendizaje como las } \\
\text { preferencias que tiene un sujeto para recibir } \\
\text { y procesar información. }\end{array}$ \\
\hline
\end{tabular}




\begin{tabular}{|c|c|c|}
\hline Modelo & Estilo de aprendizaje & Características del modelo \\
\hline Kolb & $\begin{array}{l}\text { Activo, Reflexivo, Pragmático } \\
\text { y Teórico }\end{array}$ & $\begin{array}{l}\text { Identifican dos dimensiones principales } \\
\text { del aprendizaje: la percepción y el } \\
\text { procesamiento, obteniendo que el } \\
\text { aprendizaje es el resultado de la forma de } \\
\text { percibir y de cómo procesan lo que han } \\
\text { percibido. Se plantean extremos para medir } \\
\text { los puntos fuertes y débiles. }\end{array}$ \\
\hline $\begin{array}{l}\text { Programación } \\
\text { Neurolingüística de } \\
\text { Bandler y Grinder }\end{array}$ & Visual, Auditivo y Kinestésico & $\begin{array}{l}\text { Define tres elementos como constituyentes } \\
\text { claves de la conducta humana: Visual que } \\
\text { entiende el mundo tal como lo ve. Auditivo: } \\
\text { excelente conversador. Recuerda lo que oye, } \\
\text { kinestésico: Procesa asociando al cuerpo. } \\
\text { Recuerda lo que hace. }\end{array}$ \\
\hline Hemisferios cerebrales & Lógico, Holístico, & $\begin{array}{l}\text { Permite determinar cuál de los hemisferios } \\
\text { es más dominante. }\end{array}$ \\
\hline $\begin{array}{l}\text { Inteligencias Múltiples } \\
\text { de Gardner }\end{array}$ & $\begin{array}{l}\text { Lógico-matemático, } \\
\text { Lingüístico-verbal, Corporal- } \\
\text { kinestésico, Espacial, Musical, } \\
\text { Interpersonal, Intrapersonal y } \\
\text { Naturalista }\end{array}$ & $\begin{array}{l}\text { Mencionan que la inteligencia no se puede } \\
\text { medir con un número como el coeficiente } \\
\text { intelectual. La inteligencia es la capacidad de } \\
\text { ordenar los pensamientos y coordinarlos con } \\
\text { las acciones. }\end{array}$ \\
\hline
\end{tabular}

Tabla 1 - Modelos de estilos de aprendizaje

El modelo programación neurolingüística (PNL) también conocido como modelo VAK por sus iniciales Visual, Auditivo y Kinestésico, según Bandler (1988) lo define como la mejor forma de aprender de un estudiante, filtrando la información a través de un canal predominante, facilitando la asimilación del conocimiento, esto no quiere decir que solamente se tienen que asignar tareas del estilo predominante, sino también es conveniente fortalecer los otros canales a través de diversas actividades para formar alumnos más receptivos a la adquisición de nuevos conocimientos en distintos contextos. Además, se fundamenta en tres componentes como lo menciona Díaz-Barriga (2015), primero, la programación se refiere a la aptitud humana de producir y utilizar programas de comportamiento; segundo, el componente neurológico está conformado por las percepciones sensoriales que determinan el estado emocional propio de cada persona; tercero, el componente lingüístico se refiere a la comunicación verbal y no verbal.

De acuerdo con la información mencionada anteriormente, en este trabajo se ha utilizado el modelo programación neurolingüística (PNL) para conocer los estilos de aprendizaje predominantes de los estudiantes, esto con el fin de ayudar al docente para que realice una mejor selección de la metodología empleada. De acuerdo con Gallego (2013) de esta selección metodológica dependen los resultados que se obtienen en el grupo y sobre todo se evita que se trabaje únicamente a través del canal de aprendizaje del docente o el canal que el docente considere más apropiado por sentirse más cómodo.

\subsection{Rendimiento académico}

El término de rendimiento académico se puede definir como la calificación final del estudiante (Lin, 2013). Otros autores (Vanslambrouck, 2018) mencionan que el 
rendimiento académico tiene muchas implicaciones e influyen diferentes factores determinantes tales como: el grado de satisfacción de la carrera y los factores sociodemográficos (Pauda, 2019), donde el resultado obtenido es la acción recíproca de los componentes tanto internos como externos. Es decir, depende directamente de características aptitudinales, intelectuales, la personalidad del alumno y los aspectos motivacionales durante el transcurso de los estudios universitarios.

En los últimos años, se han realizado diferentes trabajos relacionados con el desempeño académico de los estudiantes en la educación superior (Zhao, 2018), específicamente, analizan los principales factores que influyen en el rendimiento académico. Carnell et al. (2020) realizan una recopilación teórica de los principales factores que afectan al desempeño académico de los estudiantes de nivel superior, los cuales son: factores fisiológicos, pedagógicos, psicológicos y sociológicos.

Sari \& Karsen (2016) miden y evalúan el rendimiento académico a través del aprendizaje combinado efectivo implementado en una universidad privada, de acuerdo con los resultados obtenidos, se mejora la calidad del aprendizaje, sin embargo, mencionan que el aprendizaje comienza a ser eficaz a partir del compromiso institucional, esto es, la institución adquiere un fuerte compromiso para mejorar y adaptar las condiciones del aprendizaje de los estudiantes. Por otro lado, otros autores (Umek, 2015) presentan una investigación realizada en la Universidad de Ljubljana a estudiantes de administración pública, para evaluar el rendimiento académico y la satisfacción de los alumnos mediante la introducción del aprendizaje combinado.

\section{Metodología}

La utilización del método mixto, según Pereira (2011) menciona que es una excelente alternativa para abordar temáticas de investigación en el campo educativo ya que se busca la comprensión, profundización o transformación de aspectos en este campo. En este contexto, Onwuegbuzie \& Leech (2006) plantearon que las investigaciones con un diseño mixto podían ser de dos tipos: con modelo mixto: en el cual se combinan en una misma etapa o fase de investigación, tanto métodos cuantitativos, como cualitativos. Con método mixto: en cuyo caso, los métodos cuantitativos se utilizan en una etapa o fase de la investigación y los cualitativos en otra.

Después de llevar a cabo un análisis exhaustivo de la información mencionada anteriormente, en este trabajo de investigación se llevó a cabo utilizando el enfoque mixto. Se realizó una investigación cuasi experimental donde se determinaron las relaciones de causa-efecto entre dos grupos, dichos comportamientos obtenidos en el grupo control (Grupo A) fueron comparados con el grupo experimental (Grupo B). Con un enfoque mixto, longitudinal descriptivo por encuesta.

\subsection{Población y muestra}

El universo de esta investigación son los estudiantes de la Unidad Académica de Gastronomía y Nutrición Mazatlán de la Universidad Autónoma de Sinaloa (UAS), que al mes de marzo del 2020 contaba con 400 alumnos de la Licenciatura de Gastronomía; con un total de 16 grupos distribuidos de la siguiente forma: 4 grupos en primer año, 4 
grupos en segundo año, 4 grupos en tercer año y 4 grupos en cuarto año. La población con la que se trabajó fueron los estudiantes de cuarto semestre y la muestra fue por conveniencia por lo que se implementó la investigación con los estudiantes del grupo control (Grupo A: 27 alumnos) y del grupo experimental (Grupo B: 27 alumnos) que cursaron la asignatura de estadística, impartida en el cuarto semestre de la Licenciatura de Gastronomía.

El grupo control son los estudiantes que tomaron sus clases virtuales a través de la plataforma Zoom (Zoom, 2021), utilizando la plataforma Classroom (Clasroom, 2021) como herramienta para la gestión del aprendizaje y presentaciones tradicionales en Power Point para transmitir la información de las unidades de aprendizaje a los estudiantes. El grupo experimental además de las plataformas utilizadas en el grupo control, también se utilizaron diferentes herramientas tecnológicas como estrategias didácticas de acuerdo con los estilos de aprendizaje predominantes en el grupo experimental para favorecer el proceso de enseñanza aprendizaje. Algunas de las herramientas que se utilizaron fueron: Mindomo (Mindomo, 2021), Kahoot! (Kahoot!, 2021), Padlet (Padlet, 2021), Genially (Genially, 2021), Powtoon (Powtoon, 2021), Canva (Canva, 2021), Mentimeter (Mentimeter, 2021), entre otros, que sirvieron de apoyo para potencializar el conocimiento adquirido cada unidad de aprendizaje.

\subsection{Ruta metodológica}

El proceso académico se llevó a cabo de manera organizada y sistemática, primero, se solicitaron los permisos correspondientes tanto a las autoridades de la Unidad Académica como a los estudiantes participantes en esta investigación. Segundo, se aplicó un instrumento al grupo A y al grupo B llamado "Uso y aplicación de las tecnologías" de Soto (2015), el cual sirvió para analizar el contexto de esta investigación. Tercero, se procedió

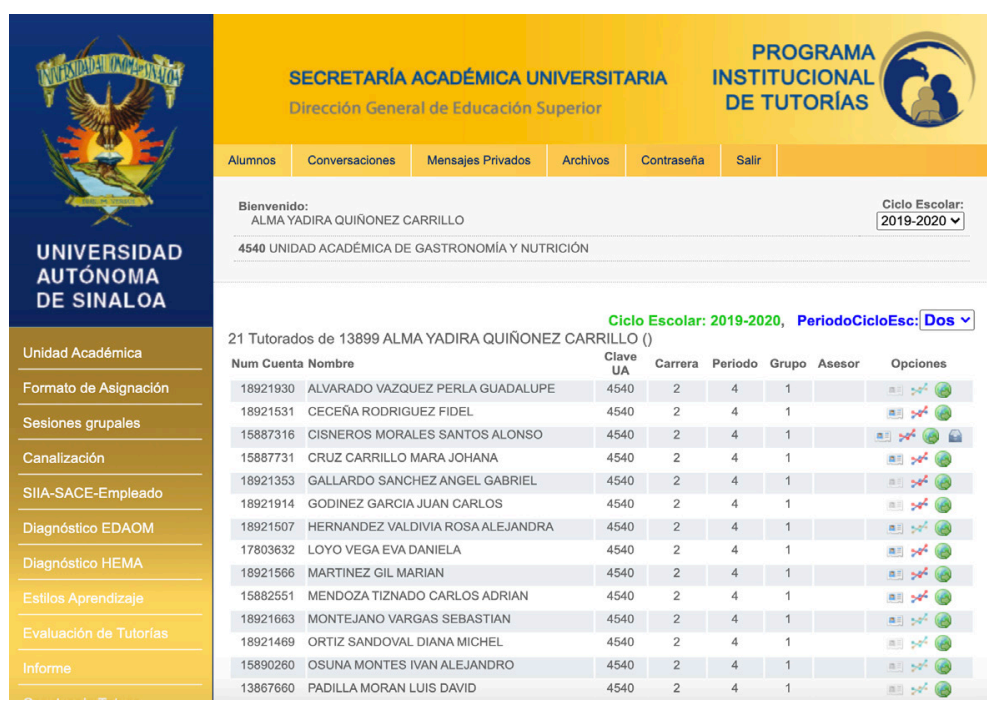

Figura 1 - Plataforma oficial del Programa Institucional de

Tutorías de la UAS, http://tutorias.uas.edu.mx/web/ 
a la aplicación del instrumento del modelo PNL desde la plataforma oficial del Programa Institucional de Tutorías de la UAS (Fig. 1), para obtener y conocer los diferentes estilos de aprendizaje predominantes de los estudiantes del grupo experimental (Grupo B). Cuarto, se llevó a cabo la aplicación de las evaluaciones tanto en el grupo control como en el experimental para evaluar los conocimientos adquiridos de la asignatura y evaluar el rendimiento académico grupal.

\section{Análisis y presentación de los resultados}

La incorporación de las tecnologías al ámbito educativo es de gran importancia para mejorar los procesos de enseñanza aprendizaje, ya que apoyan tanto al alumno como al docente en la adquisición de las competencias tecnológicas necesarias para enfrentarse al mundo laboral de actualidad. Como lo menciona Díaz-Barriga (2015), la tecnología se emplea para incrementar lo que se ha venido haciendo, pero ofreciendo una amplia posibilidad de experiencias de enseñanza, de una forma eficiente dejando atrás los ejercicios rutinarios y exámenes tradicionales. De esta forma, se logrará a través del seguimiento de pasos sistematizados los objetivos planteados para el curso, con ambientes de aprendizaje adaptados a la modalidad virtual. En este trabajo, se realizó el análisis de dos grupos que cursaron el cuarto semestre de la Licenciatura en Gastronomía, que permitieron obtener el análisis del contexto y los estilos de aprendizaje y el índice de rendimiento académico. En la tabla 2 se muestran el total de alumnos que formaron parte de la investigación.

\begin{tabular}{lccc}
\hline Grupo & Mujeres & Hombre & Total de alumnos \\
\hline $\begin{array}{l}\text { Grupo } A \\
\text { Control }\end{array}$ & 14 & 13 & 27 \\
\hline $\begin{array}{l}\text { Grupo B } \\
\text { Experimental }\end{array}$ & 11 & 16 & 27 \\
\hline
\end{tabular}

Tabla 2 - Alumnos que participaron en la investigación.

\subsection{Resultados del instrumento de contexto}

Se realizó un análisis de contexto utilizando el instrumento "Uso y aplicación de las tecnologías" de Soto (2015), con fin de extraer información pertinente para realizar esta investigación, se obtuvo información relacionada con el número de alumnos que cuentan con una computadora, celular e internet; cuántos estudiantes utilizan herramientas tecnológicas para realizar actividades escolares y con qué frecuencia. De acuerdo con los resultados obtenidos, en la figura 2 y 3 se muestra un resumen con relación al uso de la computadora, se puede observar que se obtuvieron datos relevantes que indican que la mayoría de los alumnos cuentan con herramientas tecnológicas y conexión a internet, por lo general, dichas herramientas son utilizadas diariamente para realizar trabajos escolares y acceder a redes sociales, con regularidad para descargar videos y aplicaciones. Además, se detectó como área de oportunidad que, al tener acceso de manera frecuente a internet, los alumnos, podrían aprender a optimizar los tiempos que pasan en línea para mejorar sus tareas y buscar información actualizada favoreciendo su aprendizaje. 


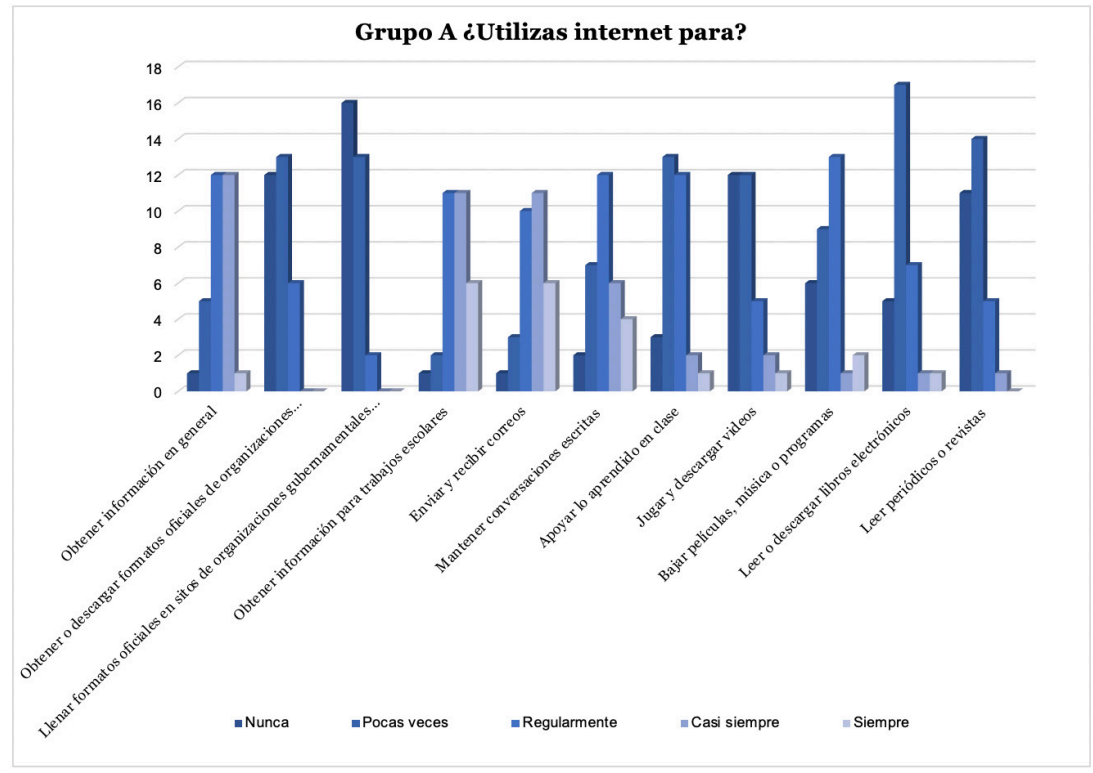

Figura 2 - Grupo A: aplicación del cuestionario uso y aplicación de las tecnologías

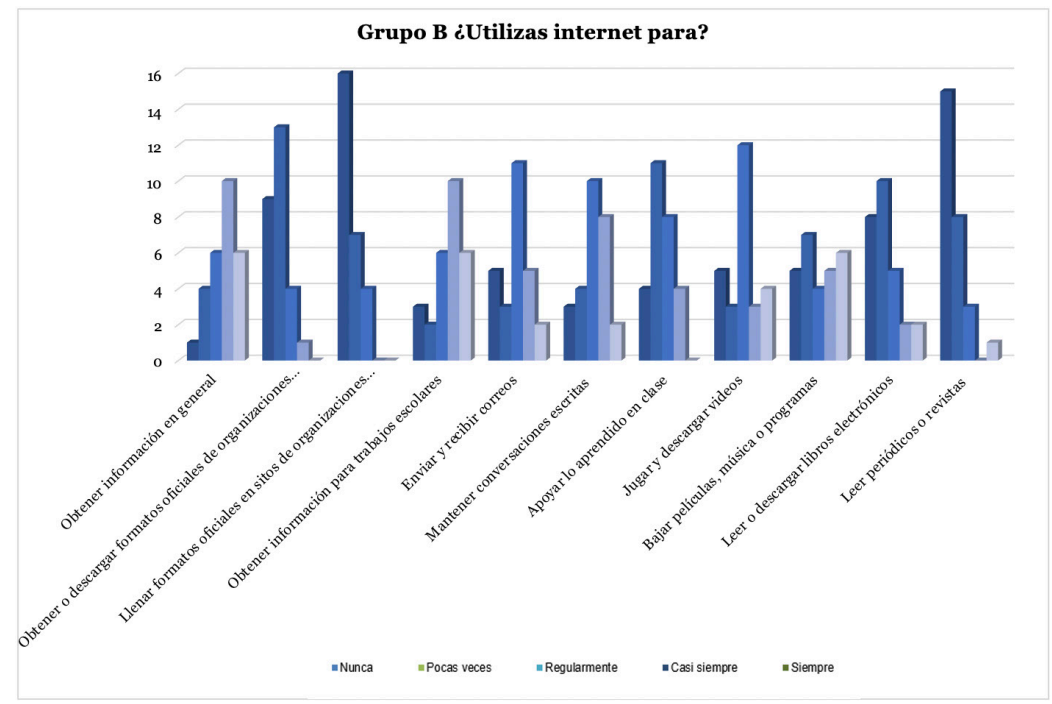

Figura 3 - Grupo B: aplicación del cuestionario uso y aplicación de las tecnologías

\subsection{Resultados del modelo PNL}

Para llevar a cabo la identificación de los estilos de aprendizaje de los estudiantes, los factores que afectan, la adquisición y la recuperación aprendida de información, se ha utilizado la plataforma institucional de tutorías desarrollada por la UAS dentro del 
Programa Institucional de Tutorías, donde además de encontrar información pública, el tutor-tutorado, tutor-asesor par, asesor par-asesorados también pueden interactuar en ese espacio los alumnos al contestar los test psicopedagógicos: como EDAOM (Estrategias de Aprendizaje y Orientación Motivacional), HEMA (Hábitos de Estudio y Motivación al Aprendizaje), CHAEA (Cuestionario Honey Alonso de Estilos de Aprendizaje) y el modelo PNL (Estilos de Aprendizaje de la Programación Neurolingüística). En este trabajo se utilizó como instrumento el modelo psicopedagógico PNL, para identificar el estilo de aprendizaje VAK de los alumnos, el cual consta de 36 preguntas de opción múltiple y se realizó directamente en la plataforma del Programa Institucional de Tutorías.

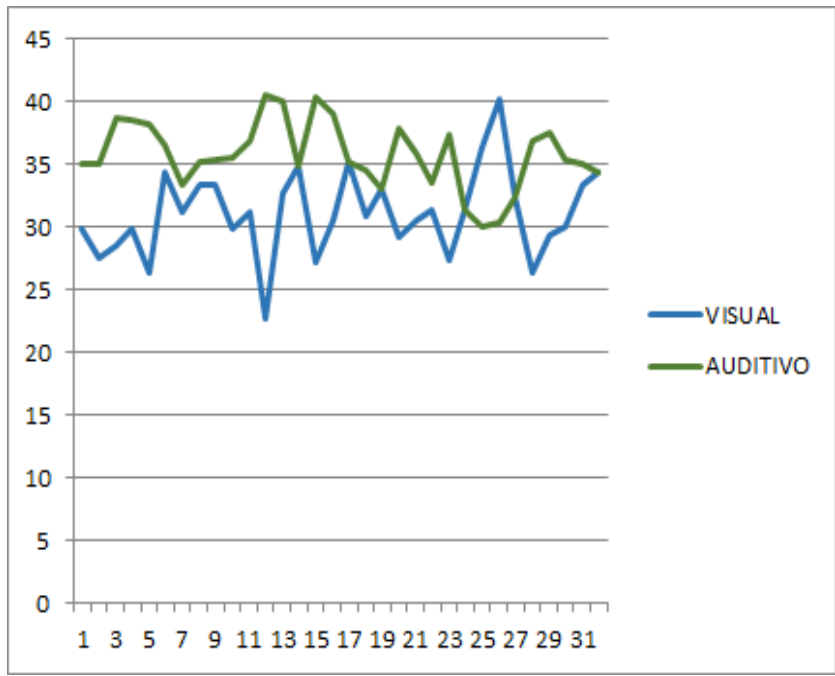

Figura 4 - Resultados del modelo PNL, comparativo de estilo visual y auditivo

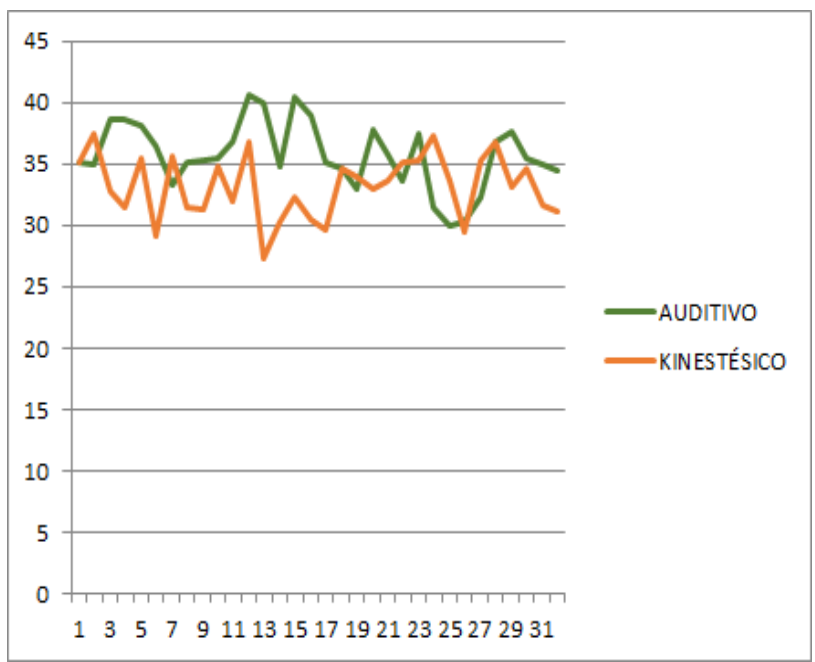

Figura 5 - Resultados del modelo PNL, comparativo del estilo auditivo y kinestésico 
De acuerdo con los resultados obtenidos, a través de la plataforma de Tutorías arrojaron que el estilo de aprendizaje que más predomina en el grupo experimental (grupo B) de la Licenciatura de Gastronomía es el visual, continuando el kinestésico y por último el auditivo. En la Figura 4, se puede observar un comparativo del estilo de aprendizaje visual con el estilo de aprendizaje kinestésico y en la Figura 5 se muestran los resultados del comparativo del estilo auditivo con el kinestésico.

\subsection{Análisis del rendimiento académico}

Una vez realizado el análisis de contexto a través del instrumento "Uso y aplicación de las tecnologías" de Soto (2015) en cada uno de los grupos y la identificación del estilo de aprendizaje predominante con el modelo PNL en el grupo experimental, se procedió a realizar el análisis del rendimiento académico que tuvieron los estudiantes durante el desarrollo de dos unidades de aprendizaje de la asignatura de estadística tanto en el grupo de control como en el grupo experimental. A continuación, en la Tabla 3 se presenta un resumen de la evaluación obtenida en cada unidad de aprendizaje para cada uno de los estudiantes del grupo control y grupo experimental.

\begin{tabular}{llllll}
\hline Grupo Control & \multicolumn{5}{l}{ Grupo Experimental } \\
\hline Estudiantes & $\begin{array}{l}\text { Unidad de } \\
\text { aprendizaje 1 }\end{array}$ & $\begin{array}{l}\text { Unidad de } \\
\text { aprendizaje } 2\end{array}$ & Estudiantes & $\begin{array}{l}\text { Unidad de } \\
\text { aprendizaje 1 }\end{array}$ & $\begin{array}{l}\text { Unidad de } \\
\text { aprendizaje 2 }\end{array}$ \\
\hline Estudiante 1 & 5.90 & 5.39 & Estudiante 1 & 8.30 & 9.33 \\
\hline Estudiante 2 & 6.45 & 8.56 & Estudiante 2 & 10.00 & 9.33 \\
\hline Estudiante 3 & 9.16 & 8.24 & Estudiante 3 & 8.30 & 10.00 \\
\hline Estudiante 4 & 7.18 & 8.23 & Estudiante 4 & 6.45 & 10.00 \\
\hline Estudiante 5 & 6.20 & 4.23 & Estudiante 5 & 8.30 & 9.33 \\
\hline Estudiante 6 & 7.89 & 4.50 & Estudiante 6 & 8.90 & 9.33 \\
\hline Estudiante 7 & 7.64 & 6.12 & Estudiante 7 & 7.68 & 9.0 \\
\hline Estudiante 8 & 8.52 & 6.23 & Estudiante 8 & 6.98 & 9.33 \\
\hline Estudiante 9 & 9.56 & 7.40 & Estudiante 9 & 9.20 & 10.00 \\
\hline Estudiante 10 & 8.15 & 8.30 & Estudiante 10 & 8.90 & 8.00 \\
\hline Estudiante 11 & 7.68 & 2.32 & Estudiante 11 & 8.80 & 9.33 \\
\hline Estudiante 12 & 7.12 & 5.16 & Estudiante 12 & 9.45 & 7.47 \\
\hline Estudiante 13 & 2.88 & 7.23 & Estudiante 13 & 9.50 & 10.00 \\
\hline Estudiante 14 & 5.72 & 5.56 & Estudiante 14 & 10.00 & 7.13 \\
\hline Estudiante 15 & 6.96 & 4.56 & Estudiante 15 & 8.90 & 7.30 \\
\hline Estudiante 16 & 7.17 & 7.82 & Estudiante 16 & 8.68 & 8.00 \\
\hline Estudiante 17 & 7.90 & 3.77 & Estudiante 17 & 4.99 & 8.67 \\
\hline Estudiante 18 & 6.00 & 8.83 & Estudiante 18 & 9.40 & 10.00 \\
\hline Estudiante 19 & 6.10 & 4.82 & Estudiante 19 & 8.79 & 8.00 \\
\hline Estudiante 20 & 4.42 & 7.59 & Estudiante 20 & 8.90 & 9.60 \\
\hline Estudiante 21 & 8.38 & 8.88 & Estudiante 21 & 5.89 & 8.88 \\
\hline & & & & & \\
\hline
\end{tabular}




\begin{tabular}{llllll}
\hline Grupo Control & \multicolumn{5}{l}{ Grupo Experimental } \\
\hline Estudiantes & $\begin{array}{l}\text { Unidad de } \\
\text { aprendizaje 1 }\end{array}$ & $\begin{array}{l}\text { Unidad de } \\
\text { aprendizaje 2 }\end{array}$ & Estudiantes & $\begin{array}{l}\text { Unidad de } \\
\text { aprendizaje 1 }\end{array}$ & $\begin{array}{l}\text { Unidad de } \\
\text { aprendizaje 2 }\end{array}$ \\
\hline Estudiante 22 & 7.09 & 9.16 & Estudiante 22 & 9.80 & 8.60 \\
\hline Estudiante 23 & 6.82 & 0.45 & Estudiante 23 & 9.60 & 9.00 \\
\hline Estudiante 24 & 7.54 & 8.26 & Estudiante 24 & 8.50 & 9.20 \\
\hline Estudiante 25 & 6.70 & 8.06 & Estudiante 25 & 10.00 & 7.40 \\
\hline Estudiante 26 & 8.26 & 5.92 & Estudiante 26 & 9.80 & 8.90 \\
\hline Estudiante 27 & 2.87 & 8.50 & Estudiante 27 & 10.00 & 10.00 \\
\hline $\begin{array}{l}\text { Promedio } \\
\text { Grupal }\end{array}$ & 6.82 & 6.52 & $\begin{array}{l}\text { Promedio } \\
\text { Grupal }\end{array}$ & 8.67 & 8.93 \\
\hline
\end{tabular}

Tabla 3 - Promedio grupal de las unidades de aprendizaje para el grupo control y para el grupo experimental.

De acuerdo con los resultados obtenidos se puede ver claramente que el rendimiento académico del grupo experimental es superior al rendimiento obtenido por el grupo control. En este contexto, para el grupo experimental el promedio de la evaluación grupal para la unidad de aprendizaje 1 es de 8.67 y 8.93 para la unidad de aprendizaje 2. Para el grupo control el promedio de la evaluación grupal para la unidad de aprendizaje 1 es de 6.82 y 6.52 para la unidad de aprendizaje 2, se puede observar que el resultado es inferior.

\section{Conclusiones}

La incorporación de las herramientas tecnológicas al ámbito educativo es de gran importancia para mejorar los procesos de enseñanza aprendizaje, debido a que, apoyan tanto al alumno como al docente para adquirir las competencias tecnológicas necesarias para enfrentarse al mundo laboral en la actualidad. En este sentido, para crear un entorno eficaz de aprendizaje en línea y enseñar cursos en línea de manera eficiente, es necesario que el profesor posea habilidades didácticas, metodológicas y tecnológicas para que el proceso de enseñanza-aprendizaje se logre satisfactoriamente. Sin embargo, la elaboración de un curso en línea no es una tarea sencilla, se requiere de componentes básicos como una planeación, para lograr de forma satisfactoria el proceso de enseñanza-aprendizaje.

Con la realización de esta investigación se obtuvieron diferentes resultados relacionados con el uso y dominio de las tecnologías y los estilos de aprendizaje del grupo control y grupo experimental. En primer lugar, se llevó a cabo la aplicación de un instrumento para analizar el uso y aplicación que le dan los alumnos a las tecnologías. Se obtuvieron datos muy interesantes como: el número de alumnos que cuentan con una computadora, celular, internet y cuántos de ellos utilizan estas herramientas tecnológicas para realizar actividades escolares y con qué frecuencia. Si bien es cierto que los estudiantes reflejan un dominio en las tecnologías, se ha identificado que solamente son utilizadas para cuestiones de redes sociales y no para académicas, en este sentido, el docente tiene la responsabilidad de guiarlos en el adecuado uso y aprovechamiento de las herramientas, 
que hoy en día, se encuentra al alcance de la mayoría de los estudiantes, con el fin de lograr vincular las TIC con el aprendizaje, de una forma organizada y lograr implementar las estrategias más adecuadas.

En segundo lugar, se llevó a cabo la aplicación del test PNL para conocer los estilos de aprendizaje que predominaba en los estudiantes. Una vez identificados los estilos de aprendizaje predominantes en cada grupo, se procedió a la realización de una adecuada planeación didáctica que permitiera trabajar y estimular todos los canales de aprendizaje, logrando una integración de las tecnologías educativas, es decir, debido a que existe una amplia gama de herramientas se pueden hacer más interactivas las actividades, como: videos, audios, películas, documentos en línea, actividades lúdicas en la web, redes sociales, entre otras. De acuerdo con los resultados obtenidos con la aplicación el modelo PNL a través de la plataforma Institucional de Tutorías de la Universidad Autónoma de Sinaloa, los estilos de aprendizaje predominantes en el grupo experimental es el visual, después kinestésico, y por último, el auditivo.

Para finalizar, es importante mencionar que toda herramienta tecnológica utilizada por el docente, esté acompañada de un previo análisis de contexto como se realizó en esta investigación, porque permite seleccionar las mejores estrategias para ser implementadas a través de la plataforma, y de esta forma, poder medir el impacto en el rendimiento académico, el cual se vio favorecido en los estudiantes que estuvieron trabajando con las diferentes herramientas tecnológicas, potencializando sus conocimientos y fortaleciendo su interacción con la tecnología. Se ha verificado que independientemente del estilo de aprendizaje que el estudiante tenga (visual, auditivo o kinestésico), al utilizar diferentes herramientas se logró tener mayor interés durante el desarrollo de las unidades de aprendizaje, y por lo tanto, los estudiantes obtuvieron un mejor rendimiento académico en la evaluación de cada unidad de aprendizaje.

\section{Referencias}

Attallah, B. (2020). Post COVID-19 Higher Education Empowered by Virtual Worlds and Applications. In Proceedings of the Seventh International Conference on Information Technology Trends (ITT), Abu Dhabi, United Arab Emirates, (pp. 161-164). IEEE Publisher.

Bandler, R., (1988). Use su cabeza para variar, submodalidades en PNL. Cuatro Vientos.

Bustos-López, M., Alor-Hernández, G., Sánchez-Cervantes, J.L., Salas-Zárate, M.P., \& Paredes-Valverde, M.A. (2018). EduRP: an Educational Resources Platform based on Opinion Mining and Semantic Web, Journal of Universal Computer Science, 24(11), 1515-1535.

Canva (2021) Diseño gráfico para todos. Disponible en: https://www.canva.com/es_mx/

Carnell, P. H., Schwab, M.C., Sochacka, N.W., \& Hunsu, N.J. (2020) Performance and perception: A preliminary examination of factors that may motivate students to bounce back. Frontiers in Education Conference (FIE), (pp. 1-4). Uppsala, Swedenç: IEEE. 
Cisco (2021). Cisco Webex Meeting:Video conferencing. Disponible en https://www.webex.com/

Google (2021). Google Classroom. Disponible en: https://classroom.google.com/

Díaz-Barriga, F. (2015). Experiencias de aprendizaje mediadas por las tecnologías digitales: pautas para docentes y diseñadores educativos. Universidad Nacional Autónoma de México.

Gallego, D. (2013). Ya he diagnosticado el estilo de aprendizaje de mis alumnos y ahora ¿qué hago. Revista de Estilos de aprendizaje, 11(12), 1-13.

Gayol de Pallán, Y.(2016) Reseña del libro Educación a distancia: teoría, investigación, diseño y evaluación. Revista Mexicana de Bachillerato a Distancia, 8(16), 159-160.

Genially (2021). Presentaciones e infografías. Disponible en: https://www.genial.ly/es

Gómez, L., Aduna, A., \& García, E. (2004). Manual de estilos de aprendizaje. México DF: Secretaría de Educación Pública. Disponible en https://drive.google.com/file/ d/oB76yT5ggiwqYNU1kZ1F3WUVOTko/edit

Google Meet. (2021). Real-time meetings, video conferencing. Disponible en https://meet.google.com/

GotoMeeting. (2021). Video conferencing. Disponible en: https://www.gotomeeting. com/en-in

Hui, T., \& Yan-Jun, Y. (2016). Knowledge Building Community in Blended Learning Environments. In Proceedings of the 8th International Conference on Information Technology in Medicine and Education, Fuzhou, pp. 581-583, IEEE Publisher.

Kahoot! (2021) Play Kahoot! Learning game. Disponible en: https://kahoot.it/

Kumar, A., Malhotra, S., Katoch, A., Sarathkar, A., \& Manocha, A. (2020). Webinars: An assistive tool used by higher education educators during Covid19 case study. In Proceedings of the 12th International Conference on Computational Intelligence and Communication Networks (CICN), Bhimtal, India, (pp. 1-6). IEEE Publisher.

Lekka, A., Tsironi, M., \& Pange, J. (2015). New trends of information and communication technologies in education. In IEEE International Conference on Interactive Mobile Communication Technologies and Learning, Thessaloniki, Greece, (pp. 389-389). https://doi.org/10.1109/IMCTL.2015.7359626.

Lillejord, S., Borte, K., Nesje, K., \& Ruu, E. (2018). Learning and teaching with technology in higher education - a systematic review. Oslo: Knowledge Centre for Education.

Lin, C.C., \& Chiu, C. H. (2013). Correlation between Course Tracking Variables and Academic Performance in Blended Online Courses. In Proceedings of the $13^{\text {th }}$ International Conference on Advanced Learning Technologies, Beijing, China, (pp. 184-188). IEEE Publisher. 
Lizarraga, C., Quiñonez, Y., Aguayo, R., \& Peraza, J. (2017). Incorporation of robots in the teaching-learning process for the different educational levels. In Proceedings of the 9th International Conference on Education and New Learning Technologies, Barcelona, Spain, pp. 5459-5457, Iated Digital Library.

Mentimeter (2021). Interactive presentation software. Disponible en: https://www.mentimeter.com/

Microsoft Team (2021). Video conferencing. Disponible en: https://www.microsoft.com/ es-mx/microsoft-teams/online-meetings

Mindomo (2021). Mapas mentales y conceptuales. Disponible en: https://www.mindomo.com/es/

Minetti, M. L. (2015). ¿Enseñar o aprender? Escritos en la facultad, 11(109), 55-56.

Onwuegbuzie, A. J., \& Leech, N. L. (2006). Linking research questions to mixed methods data analysis procedures 1. The Qualitative Report, 11(3), 474-498. https://doi.org/10.46743/2160-3715/2006.1663

Padlet (2021). Tableros colaborativos. Disponible en: https://es.padlet.com/

Pantoja, M.A., Duque, L.I., \& Correa, J.S. (2013) Modelos de estilos de aprendizaje: una actualización para su revisión y análisis. Revista Colombiana de Educación, (64), 79-105. Disponible en: https://www.redalyc.org/articulo.oa?id=413634076004

Padua Rodríguez, L.M. (2019). Factores individuales y familiares asociados al bajo rendimiento académico en estudiantes universitarios. Revista mexicana de investigación educativa, 24(80), 173-195. Disponible en: http://www.scielo.org. $\mathrm{mx} /$ scielo.php?script=sci_arttext\&pid=S1405-66662019000100173\&lng=es\&tlng $=$ es.

Pereira Pérez, Z. (2011). Los diseños de método mixto en la investigación en educación: Una experiencia concreta. Revista Electrónica Educare, 15(1), 15-29. Disponible en: https://www.redalyc.org/articulo.oa?id=194118804003

Powtoon (2021). Make video and animations online. Disponible en: https://www.powtoon.com/

Quiñonez, Y., Luzardo, G., \& Granda, R. (2016). Implementación de un sistema multitáctil en ambientes educativos para promover y facilitar la evaluación del trabajo colaborativo en el aula. RISTI - Revista Ibérica de Sistemas e Tecnologias de Informação, (17), 66-79. https://doi.org/10.17013/risti.17.66-79

Sari, S., \& Karsen, M. (2016). An empirical study on blended learning to improve quality of learning in higher education. In IEEE International Conference on Information Management and Technology, pp. 235-240.

Seitebaleng, S. D. (2018). Information and communication technology use in higher education: Perspectives from faculty. International Journal of Education and Development using Information and Communication Technology, 14(2), 121-166. 
Shuell, T. J. (1986). Cognitive conceptions of learning. Review of Educational Research, 56, 411-436. http://dx.doi.org/10.3102/00346543056004411

Siemens, G., \& Fonseca, D. (2007). Conectivismo: Una teoría de aprendizaje para la era digital. Philosophy.

Skinner, B. F., \& Ardila, R. (1977). Sobre el conductismo. Fontanella.

Soto, M.G. (2015). Tecnologías de Información y Comunicación en los procesos educativos: Sus representaciones sociales desde el docente. Revista Educativa Hekademos, (17), 23-30.

Srivastava, T., Waghmare, L.S., Jagzape, A., Rawekar, A., Quazi, N., \& Mishra, V.P. (2014). Role of information communication technology in higher education: learners perspective in rural medical schools. Journal of clinical and diagnostic research, 8(6), XCo1-XCo6. https://doi.org/10.7860/JCDR/2014/8371.4448

Umek, L., Keric, D., Aristovnik, A., \& Tomaevic, N. (2015). Implications of Blended Learning on Students' Performance in Public Administration Education. In IEEE nternational Conference on u- and e-Service, Science and Technology, pp. 39-44.

Vanslambrouck, S., Zhu, C., Lombaerts, K., Philipsen, B., \& Tondeur, J. (2018). Students' motivation and subjective task value of participating in online and blended learning environments. The Internet and Higher Education, (36), 33-40.

Zambrano, L.L. (2020) Uso de la Tecnología de la Información y Comunicación en educación virtual y su correlación con la Inteligencia Emocional de docentes en el Ecuador en contexto COVID-19. RISTI - Revista Ibérica de Sistemas e Tecnologias de Informação, (40), 31-44. https://doi.org/10.17013/risti.40.31-44k

Zhao, H., Guo, Y., Wang, K., \& Lu, M. (2018). The Determinants of Students' Academic Achievement in B-Leaming Environment: An Empirical Investigation, In 13th International Conference on Computer Science \& Education (ICCSE), Colombo, pp. 1-5, https://doi.org/10.1109/ICCSE.2018.8468828.

Zoom (2021). Video conferencing, Web conferencing, Webinnars. Disponible en: https://zoom.us/meetings 